\title{
Microarray-based method for detection of unknown genetic modifications
}

\author{
Torstein Tengs ${ }^{1}$, Anja B Kristoffersen ${ }^{2,3}$, Knut G Berdal ${ }^{1}$, Tage Thorstensen ${ }^{4}$, \\ Melinka A Butenko ${ }^{4}$, Håvard Nesvold ${ }^{1,3}$ and Arne Holst-Jensen*1
}

\begin{abstract}
Address: ${ }^{1}$ National Veterinary Institute, Section of Feed and Food Microbiology, PO Box 8156 Dep, 0033 Oslo, Norway, ${ }^{2}$ National Veterinary Institute, Section of Epidemiology, PO Box 8156 Dep, 0033 Oslo, Norway, ${ }^{3}$ University of Oslo, Department of Informatics, PO Box 1080 , Blindern, 0316 Oslo, Norway and ${ }^{4}$ University of Oslo, Department of Molecular Biosciences, PO Box 1041, Blindern, 0316 Oslo, Norway

Email: Torstein Tengs - torstein.tengs@vetinst.no; Anja B Kristoffersen - anja.kristoffersen@vetinst.no; Knut G Berdal - knut.berdal@vetinst.no; Tage Thorstensen - tage.thorstensen@imbv.uio.no; Melinka A Butenko - m.a.butenko@imbv.uio.no; Håvard Nesvold - nesvold@gmail.com; Arne Holst-Jensen* - arne.holst-jensen@vetinst.no

* Corresponding author
\end{abstract}

Published: 18 December 2007

BMC Biotechnology 2007, 7:9| doi:|0.||86/|472-6750-7-9|
Received: 24 May 2007

Accepted: 18 December 2007

This article is available from: http://www.biomedcentral.com/1472-6750/7/91

(C) 2007 Tengs et al; licensee BioMed Central Ltd.

This is an Open Access article distributed under the terms of the Creative Commons Attribution License (http://creativecommons.org/licenses/by/2.0), which permits unrestricted use, distribution, and reproduction in any medium, provided the original work is properly cited.

\begin{abstract}
Background: Due to the increased use of genetic modifications in crop improvement, there is a need to develop effective methods for the detection of both known and unknown transgene constructs in plants. We have developed a strategy for detection and characterization of unknown genetic modifications and we present a proof of concept for this method using Arabidopsis thaliana and Oryza sativa (rice). The approach relies on direct hybridization of total genomic DNA to high density microarrays designed to have probes tiled throughout a set of reference sequences.

Results: We show that by using arrays with 25 basepair probes covering both strands of a set of 235 vectors ( 2 million basepairs) we can detect transgene sequences in transformed lines of $A$. thaliana and rice without prior knowledge about the transformation vectors or the T-DNA constructs used to generate the studied plants.

Conclusion: The approach should allow the user to detect the presence of transgene sequences and get sufficient information for further characterization of unknown genetic constructs in plants. The only requirements are access to a small amount of pure transgene plant material, that the genetic construct in question is above a certain size (here $\geq 140$ basepairs) and that parts of the construct shows some degree of sequence similarity with published genetic elements.
\end{abstract}

\section{Background}

Since the first genetically modified (GM) plants were authorized for release and commercial food production in the mid 1990ies, the global GM acreage has increased rapidly every year. For example $57 \%$ of all soybean and $25 \%$ of all maize grown on the world market in 2006 was GM [1]. The diversity of food crop species and traits introduced is also rapidly increasing, and with the prospective use of food crop plants in for instance production of pharmaceuticals and biofuels, the risk is also increasing for introduction of unauthorized GM material into the foodchain or release of unauthorized GM plants into the environment. Recent examples of such introduction are the presence of event Bt10 in US maize and LL601 rice in US rice exported to Europe $[2,3]$. International and national regulations require that distribution and release of GM 
organisms (GMOs) is under strict control and surveillance, and that GMOs are only authorized on the basis that they do not pose a risk to human or animal health or the environment [4]. With the increased global focus on biosafety, and the realistic scenarios of unintended release of unauthorized and potentially unknown nature of the GM materials, there is a need for availability of technology that rapidly can detect and provide information about a possible unknown GMO.

Genetic modifications of plants (and other organisms) involve the stable introduction of a novel genetic construct (usually carried by a vector) into a target organism's genome. In plants, the most popular traits introduced include herbicide tolerance, insect resistance and ripening delay [5]. In addition to the particular gene associated with the trait of interest, other genetic elements are also transferred to the target plant during a transformation event. For correct expression, promoters such as the cauliflower mosaic virus (CaMV) 355 promoter (P35S) are needed. Among the most popular terminators/polyadenylation signals are the nopaline synthase (NOS) terminator from Agrobacterium tumefaciens and the CaMV $35 S$ polyadenylation signal (T35S). In addition, selection markers, polylinkers and transit peptides are often included in GM constructs. Depending on the transformation technique used, as well as other factors, additional parts of the carrier vector may also be co-transformed into the target genome.

The most widely used methods for detection of genetic modifications in plants rely on real-time PCR. Primers can be designed either to be used as screening tools where consensus primers target a commonly used GM element, gene-specific primers can be used to detect the presence of an introduced trait gene, construct specific assays can be used to specifically target a particular configuration of GM elements, or host/transgene junction-specific primers can be designed to make an assay event-specific [6]. In addition to fluorescent real-time PCR, some methods have also been described where amplified products are labeled and hybridized to arrays [7-10] and multiplex setups have been suggested $[11,12]$. Still, singleplex PCR remains the most commonly used strategy, and if the genetic modification in question is unknown or poorly characterized, multiple individual PCR experiments are set up. Each PCR assay is designed to target a particular sequence, and multiple assays (and controls) have to be performed in order to check for the presence of different genetic constructs.

We have developed a high resolution microarray-based method for the detection of both known and unknown genetic modifications in plants. The method is PCR-independent, applies direct hybridization of total genomic DNA and takes advantage of the high degree of recycling and sequence similarity between elements commonly used in genetic modifications in plants. Using custom designed microarrays, we have analyzed genetically modified lines of Arabidopsis thaliana and Oryza sativa (rice) as model systems. We show that without prior knowledge about the transgene sequence in question, fragments $(\geq$ $140 \mathrm{bp}$ ) of the elements used in the genetic transformation can be detected.

\section{Results}

NimbleExpress arrays (Affymetrix, Santa Clara, CA, USA) were designed to have 25 basepair probes tiled throughout 235 vector sequences downloaded from GenBank (a total of approximately 2 megabases of sequence; for list of sequences used in design [see Additional file 1]. In order to achieve robust signal intensities from positive probes, we decided to use the highest possible quantity (mass) of DNA given the volume of hybridization mixture that could be injected into the Affymetrix array cartridge. Ninety $\mu \mathrm{g}$ seemed to be the upper limit (more DNA resulted in poor array performance), and this corresponds to roughly 1 fmole haploid A. thaliana genomes and 267 amoles of haploid rice genomes.

Six arrays were run, representing two transgene A. thaliana lines, two experimental duplicates of wildtype A. thaliana, as well as one transgene and one wildtype rice line. Average signal intensities for the array probes were 196.92, 209.71, 203.98, 192.95, 260.43 and 226.06 (ida, SALK, A. thaliana wildtype replicates, wildtype and CecA rice). After normalization of the arrays, signal/background ratios were calculated for all probes using the average signals from the wildtype replicates as an estimate for the level of probe signal background (Table 1). The complete set of signal data is available upon request.

The analysis did not yield any false positives looking at wildtype vs. wildtype A. thaliana DNA (Table 1). As expected for the other experiments, a large fraction of the sequence windows detected formed small sets of overlapping and nearly identical sequences. The initial analysis gave 394 positive windows for SALK/WT, 145 for $i d a / W T$ and 152 for CecA/WT (Table 1). After sequence filtering (see description in Materials and Methods) of data from the ida experiment, we were left with 11 unique sequences. In the SALK experiment, 15 sequences were reported and analysis of the rice DNA yielded 8 unique windows. Detected windows ranged in size from 147 to 325 bases, representing 32 to 63 probes (Table 1; for a complete list of detected sequence windows, [see Additional file 2]). All of the high ranking matches could easily be matched back to their corresponding T-DNA loci (Figure 1). For SALK, the highest ranking windows were dominated by elements containing the NOS terminator. This element is present twice in the SALK insert and this might 
Table I: Array performance.

\begin{tabular}{|c|c|c|c|c|}
\hline & & A. thaliana & & Oryza sativa \\
\hline & ida/WT & SALK/WT & WT/WT & CecA/WT \\
\hline Mean signal ratio & 0.96 & 0.95 & 0.98 & 1.09 \\
\hline Signal ratio range & $0.049-9.64$ & $0.13-18.45$ & $0.085-3.29$ & $0.24-23.58$ \\
\hline Standard deviation (signal ratio) & 0.35 & 0.67 & 0.16 & 0.72 \\
\hline $\begin{array}{l}\text { Total number of positive probes (percent of total number } \\
\text { of probes) }\end{array}$ & $854(2.29 \%)$ & $1026(2.75 \%)$ & $1120(3.00 \%)$ & $845(2.27 \%)$ \\
\hline Number of probes for target sequence & 1252 & 2056 & - & 910 \\
\hline True positive probes (target sequence) & 605 & 745 & - & 322 \\
\hline False positives (percent of total number of probes) & $249(0.69 \%)$ & $28 I(0.80 \%)$ & $1120(3.00 \%)$ & $523(1.40 \%)$ \\
\hline Number of windows detected & 145 & 394 & 0 & 152 \\
\hline Number of windows after filtering & II & 15 & - & 8 \\
\hline Number of probes per window & $33-63$ & $32-59$ & - & $32-49$ \\
\hline Length of windows & $176-325$ & $|67-3| 2$ & - & $147-255$ \\
\hline
\end{tabular}

lead to a bias due to stronger signals from these probes. This same phenomenon was observed for the double enhancer version of the CaMV $35 S$ promoter in the CecA rice. All of the sequences detected matched close to $100 \%$ perfectly with the target sequences except for some single basepair differences (Figure 1).

Subsequent Southern blotting of DNA from the SALK line using probes targeting the insertion cassette indicated that this was indeed a single copy line, and analysis of sequence data showed that the DNA transferred during the transformation appeared to be limited to the T-DNA region (data not shown). Neither the T-DNA tagging vector pMHA2 [13] used to generate the ida plant nor the SALK insertion line T-DNA (from the pBIN-pROK2 vector; [14]) were part of our set of CaMV P35S-containing vectors used for array design. The rice construct contained a large fragment derived from the PCAMBIA-1300 vector, which was included in our target sequence compilation.

\section{Discussion}

The recent increase in feature density and lowered cost associated with the use of microarrays has prompted several groups to develop whole-genome based protocols for characterization of eukaryotic genomic content $[15,16]$. Most of these groups have relied on currently available (expression) microarrays. Using a single color system, we decided to see if this approach would work using custom designed arrays and unfiltered probes tiled throughout a set of reference sequences in the context of genetically modified plants.

Neither of the transgene plant lines tested were transformed using constructs where complete sequence information had been submitted to GenBank, so in the context of this study they were treated as unknown genetic modifications. But, the method does make some assumptions about the genetic elements that can be uncovered. For example, with the applied minimum window size of 30 probes, the transgene sequence in question must contain at least one element of a significant size (here $\geq 140 \mathrm{bp}$ ) that has a reasonably high degree of sequence similarity with one or more of the elements that were included in our target sequence compilation. In this sense, the method is not able to detect all 'truly unknown GMOs'. An array-based method that makes fewer assumptions has been suggested by our group previously [17], but currently no methods (besides perhaps whole genome sequencing) can be said to guarantee the accurate detection of a genetic modification without making any assumptions. Window sizes of 10 and 20 probes were also tested but these yielded too many false positives. The absolute optimal combination of window size and match cutoff (here $70 \%$ of the probes in a window) was not determined in the present study.

In our array design, more than 2 million bases of sequence were used to design the overlapping probes. The fact that less than 40,000 probes were sufficient to get a degree of coverage of on average one probe per five bases (both strands) gives an indication of the level of conservation and reuse when it comes to the elements used in designing genetic constructs for plant transformations. Although a novel, unknown GMO might contain a trait gene not included in a reference sequence database, it is highly likely that other elements, such as promoters, selection markers, terminators etc. will be used that have been described in the literature. The entire GenBank vector sequence collection now includes information from about 3,600 entries (> 20 megabases of sequence), but this only constitutes about $5.24 \times 10^{6}$ unique 25 basepair sequences (both strands of the 3,600 sequences). The highest number of features currently synthesized on commercially available Affymetrix-type arrays is $>6 \times 10^{6}(500$ 
ida
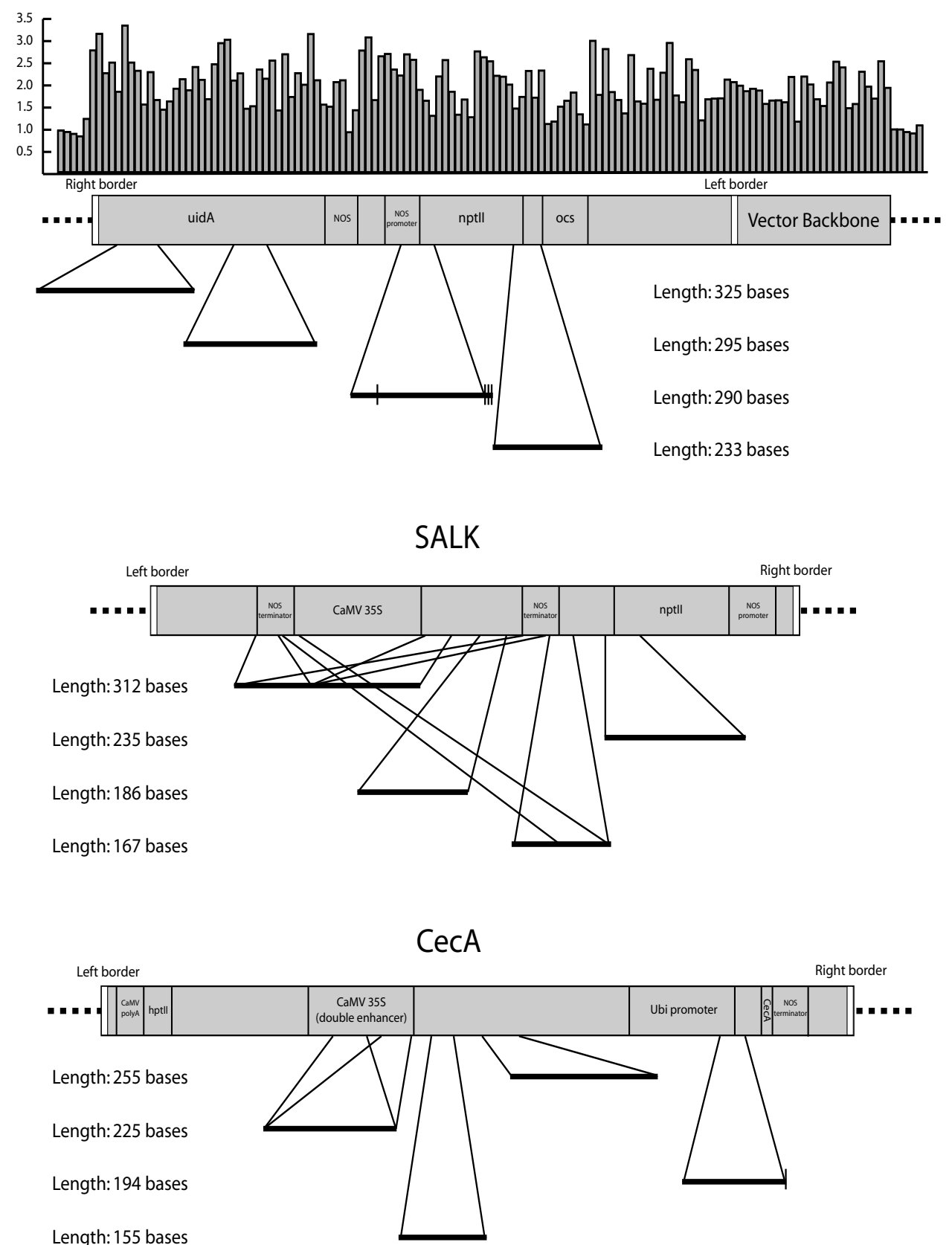

\section{Figure I}

Sequences detected by the arrays. The detected sequence windows were ranked according to the number of probes that defined them and the four highest ranked windows with non-overlapping hits to the T-DNA sequences are shown (for a complete list of positive windows, [see Additional file 2]. The scale for the detected windows is $4 \times$ the scale for the T-DNA constructs. Hatch marks correspond to point mutations. The bar diagram showing signal/noise ratio for matching probes (averaged across sets of 10 probes) has been included for the ida dataset as an example. The I,239 bp vector backbone fragment in ida has been described previously [28]. NOS - nopaline synthase. oct - octopine synthase. nptll - neomycin phosphotransferase II. CaMV 35S - cauliflower mosaic virus $35 \mathrm{~S}$ promoter. uidA - beta glucuronidase. CaMV polyA - cauliflower mosaic virus 3' UTR polyadenylation signal. hptll - hygromycin phosphotransferase. CaMV 35S (double enhancer) - cauliflower mosaic virus $35 \mathrm{~S}$ promoter, double enhancer version. Ubi - maize ubiquitin. CecA - cecropin A. 
K Mapping Array Set), so it should be possible to make an array with any degree of probe tiling scheme throughout both DNA strands of any set of cloning vectors in GenBank.

Even though our probes were designed using a set of 235 sequences, our window-based approach for detecting GM elements could be used with reference to any set of relevant sequences. We have only screened our results using the same reference sequences we used for the array design, but as long as the probe coverage is reasonably high, data from an array design such as the one described in this study may be compared against a much broader sampling of transgene sequences.

With the current design, it cannot be assumed that very long stretches of target sequence can be detected in their entirety (neither can it be assumed that the exact construct structure one wants to detect is part of the sequence compilation used to match probe signal intensities against). For instance, the vector used in the generation of T-DNA SALK insertion lines was not included in the target sequence compilation used for our array design, but it has a T-DNA sequence with a stretch of 3,475 bases with $100 \%$ match to one of the vectors in our target sequence compilation (binary vector pBI121; GenBank accession number $\underline{\mathrm{AF} 485783}$ ). Still, we were not able to detect a continuous window for this part of the T-DNA construct. In our experiments, 35-48\% percent of the probes that should yield a positive signal were scored as true positives (from 322 out of 910 true positive probes for rice to 605 out of 1252 for SALK; Table 1). Poor performance of a fraction of the probes was not unexpected since they were designed without in silico filtering $[18,19]$ or empirical testing. It is, however, also important to remember that the cutoff value for scoring a probe as positive using our strategy relies on the standard deviation of the signal/ noise ratios. The standard deviation will be affected by the number of potentially positive probes in an experiment. If a large number of probes on the array match the GMO analyzed, the standard deviation is likely to increase. So, the number of probes that are scored as positives is linked to the size of the GM element in question. An A. thaliana line transformed with for instance a truncated version of the ida construct would probably have led to a smaller standard deviation, and would have given a different set of positive probes and probe windows. So, the sensitivity of the method is likely to increase when the number of potentially positive probes decreases. While at first glance, the results for $\mathrm{WT} / \mathrm{WT}$ in Table 1 seem to indicate that without true positives in the sample, the method becomes oversensitive ( $3 \%$ false positive probes), the windowbased approach readily discriminates between false positive individual probes and false positive windows (no windows were detected for WT/WT).
Thus, the presented method should be seen more as a tool for detecting discrete transgene elements than a protocol for detailed characterization of genetically modified plants. By using sequence similarity search tools such as BLAST [20], it is fairly straightforward to at least partly annotate the positive windows. As a starting point for further characterization of the transgene sequence detected, we recommend using defined genetic loci within the hits rather than the raw sequences. The most commonly used protocols for characterization of GM events rely on anchor-PCR followed DNA sequencing of amplified products [21,22], see also [23] and the sequences detected by our method should be sufficient for initiating a more detailed study using these standard approaches.

The general concept described herein should work well for organisms other than the plant lines described herein, albeit it is likely that a significantly larger genome (human or wheat, for instance) size will decrease the signal/noise ratio using our current protocol for DNA labeling and hybridization. Given the upper limit on the amount of DNA that can be hybridized, a larger genome will give a smaller number of target DNA copies per feature on the array. One way to avoid this problem might be to implement a more sensitive labeling strategy, using for instance quantum dot technology [24]. An alternative approach would be to co-hybridize differentially labeled control and sample DNA and use the signal ratios directly.

\section{Conclusion}

The whole genome based concept described herein should be useful for both detection and characterization of known as well as unknown genetic modifications in plants. The method requires access to a small amount of GMO material of high purity, but the only other limitations are availability of sequence data from GMO constructs and the minimal size of the transgene sequence to be detected. It is expected that the upper limit for number of features that can be fitted onto an array will continue to increase, and combined with decreased array cost, we believe that unbiased array-based detection of GM constructs will be a helpful tool not only for research on plants, but for genetically modified organisms in general.

\section{Methods \\ Array design}

For the array design, sequences were chosen that contained (parts of) CaMV P35S based on sequence similarity searches such as BLAST [20]. CaMV P35S is among the most widely used genetic elements in genetic modifications of plants [25-27], and its presence was thus used as a proxy to identify cloning vectors and other constructs likely to be relevant for plant transformations. To achieve maximum probe density using a minimum number of probes, the following probe selection strategy was used: 
first, all 25 basepair fragments were sequentially extracted from the set of 235 sequences. A corresponding set of reverse-complementary probes was also generated. From these ordered lists, all probes containing ambiguous bases were excluded and duplicate probes removed. Finally, every $10^{\text {th }}$ probe was extracted from the probe database. In the array design, the majority of the probes thus had 15 bases overlaps with neighboring probes on both strands of the target sequences. Due to the high degree of similarity between the elements commonly used in vector sequences, 37,257 probes were sufficient to achieve this coverage.

For general assessment of array performance, positive and negative control probes were also included. The array design included 1,000 positive control probes picked from the Affymetrix A. thaliana expression array (GeneChip Arabidopsis ATH1 Genome Array) as well as 1000 positive control probes picked from the GeneChip Rice Genome Array (Affymetrix). The positive control probes were selected so that they corresponded to single copy loci in the respective plant genomes. In addition, 1,000 negative control probes were designed (random 25 bp sequences with no matches in the A. thaliana or rice nuclear, chloroplast or mitochondrial genomes).

\section{DNA extraction and whole genome amplification}

Wildtype A. thaliana ecotype Columbia (Col-0) plants, the inflorescence deficient in abscission (ida) mutant [28], and the SALK_128444 line purchased from the Arabidopsis Biological Resource Center (Columbus, OH, USA), were all grown under long day greenhouse conditions at $20^{\circ} \mathrm{C}$. DNA was extracted using the method described by Dellaporta et al.[29] and resuspended in molecular grade water.

For the rice material, an approach was developed to avoid the requirement of access to the relatively large amounts of genomic DNA described for the A. thaliana protocol. DNA was isolated from $30 \mathrm{mg}$ of wildtype Mediterranean elite japonica rice (Oryza sativa L.) cultivar Senia and rice transformed with the cecropin A ( $\mathrm{CecA}$ ) gene [30] using a CTAB based protocol [31]. The DNA was used to perform whole genome amplification (WGA) using the REPLI-g whole genome amplification kit (QIAGEN AB, Sweden) according to the manufacturer's recommendations. The WGA products were extracted using phenol:chloroform:isoamyl alcohol 25:24:1 (saturated with $10 \mathrm{mM}$ Tris, pH 8.0, 1 mM EDTA; Sigma-Aldrich Norway AS, Oslo, Norway) and ammonium acetate/ethanol precipitated. After resuspension in distilled water, the material was processed the same way as the DNA isolated from A. thaliana.

\section{DNA fragmentation, labeling and hybridization}

For each experiment, $90 \mu \mathrm{g}$ of DNA was fragmented using 4 units ( 8 units for the WGA DNA) of DNase I (New England Biolabs, Ipswich, MA, USA) in a total volume of 500 $\mu \mathrm{l} 1 \times$ DNase I Reaction Buffer (New England Biolabs). The fragmentation reaction was incubated for 5 minutes at $37^{\circ} \mathrm{C}$, followed by 10 minutes at $95^{\circ} \mathrm{C}$ to inactivate the enzyme. The fragmented DNA was phenol:chloroform:isoamyl alcohol extracted and ammonium acetate/ ethanol precipitated and end labeled using 90 units of terminal deoxynucleotidyl transferase (Promega, Madison, WI, USA) and 5 nmoles of biotin-16-2',3'-dideoxy-uridine-5'-triphosphate (biotin-16-ddUTP; Roche Diagnostics Norge AS, Oslo, Norway). The labeling reaction was performed in $300 \mu \mathrm{l}$ reaction volume with $1 \times$ Terminal Deoxynucleotidyl Transferase Buffer (Roche Diagnostics Norge AS) for two hours at $37^{\circ} \mathrm{C}$ followed by 10 minutes at $95^{\circ} \mathrm{C}$ (inactivation of the enzyme). The DNA was again phenol:chloroform:isoamyl alcohol extracted and ammonium acetate/ethanol precipitated.

Several different array hybridization, washing and staining protocols were tested for the arrays. Initially, a hybridization buffer containing tetramethylammonium (TMA) was used. The presence of high concentration of TMA has been shown to reduce bias in hybridization efficiency due to differences in GC content [32], and since the probes on our arrays were not filtered according to base composition (or any other criteria), we believed that such an experimental setup could prove to be the most suitable. However, we concluded that the protocol recommended by Affymetrix for hybridization of PCR amplified chromatin immunoprecipitation products (Affymetrix Chromatin Immunoprecipitation Assay protocol) gave the highest average positive control/negative control probe signal ratio. In order to mimic these hybridization reaction conditions, the pellet was thus resuspended in $4.3 \mu \mathrm{l} 10 \times$ Fragmentation Buffer (Affymetrix), $12 \mu \mathrm{l} 5 \times$ TdT Buffer (Affymetrix), $3.7 \mu$ l Control Oligo B2 (Affymetrix), 15.4 $\mu \mathrm{l}$ DMSO, $110 \mu \mathrm{l} 2 \times$ Hybridization Buffer (for recipe, see Affymetrix manual for Affymetrix Chromatin Immunoprecipitation Assay; revision 3, Appendix B) and $74.6 \mu \mathrm{l}$ molecular grade water (total volume: $220 \mu \mathrm{l}$ ). Arrays were otherwise processed and handled as described in the Affymetrix protocol for Affymetrix Chromatin Immunoprecipitation Assays, but the array cartridge rotation speed during hybridization was reduced from $60 \mathrm{rpm}$ to $20 \mathrm{rpm}$ to facilitate efficient mixing of the viscous hybridization mix (due to the relatively high amount of DNA used).

\section{Data analysis}

The sequence for the SALK T-DNA insert was downloaded from the Salk Institute Genomic Analysis Laboratory web page [33] and the ida sequence for the pMHA2 T-DNA was kindly provided by Dr. Abul Mandal (University of 
Skövde). It is noteworthy that the ida construct does not contain the CaMV P35S element that was used as a proxy for the initial stage of the array design (Figure 1). The sequence for the CecA rice line was derived from published information (see [28] for references). Sequences were compared to the T-DNA insert by using the tool 'bl2seq' [34].

For the analysis, raw signal intensities were exported from the GeneChip Operating Software (Version 1.2; Affymetrix) using the software tool IntensityExporter [35]. Average background signal for all vector probes was calculated using two experimental duplicates of wildtype A. thaliana DNA. Assuming that the fraction of probes corresponding to true positives in any given experiment will be very low, signal intensities for all arrays were normalized to give the same average vector probe signal (absolute value: 200). The probes and their corresponding signal intensities were mapped back to their positions in the 235 vectors used in the array design. For all probe positions, signal/background ratio was calculated, and these ratios were screened using a window-based approach. A probe was considered positive if the signal/background ratio was higher than the mean ratio plus two standard deviations, and a minimum window size of 30 probes was chosen. This window was slid through all the vector sequences, and was scored as positive if more than $70 \%$ ( 21 or more) of the probes were positive. When a positive window was detected, the window was gradually expanded to include more downstream probes. This expansion was continued until the fraction of positive probes fell below $70 \%$. The sequence windows were subsequently filtered by removing windows completely encompassed by other windows, and sequence fragments were trimmed to remove flanking regions corresponding to negative probes. Remaining sequences were tabulated and ranked according to the number of probes they encompassed.

\section{Authors' contributions}

TTengs conceptualized the project, did all the hybridization experiments and wrote the final version of the paper. ABK performed the data analyses, KGB and AH-J contributed to experimental design and data interpretation. TThorstensen did the Southern blotting and characterization of the SALK A. thaliana line and MAB provided data for the ida line. HN wrote the software for probe selection. All authors read and approved the final manuscript.

\section{Additional material}

\author{
Additional file 1 \\ Vector sequence list. A listing of the 235 vector sequences downloaded \\ from GenBank and used in the design of probes on the array. \\ Click here for file \\ [http://www.biomedcentral.com/content/supplementary/1472- \\ 6750-7-91-S1.doc]

\section{Additional file 2} \\ Detected sequence windows. A complete description of the detected \\ sequence windows, for all three T-DNA inserts. \\ Click here for file \\ [http://www.biomedcentral.com/content/supplementary/1472- \\ 6750-7-91-S2.xls]
}

\section{Acknowledgements}

The authors would like to thank Roy Falleth for help with DNA extractions, Siv $\mathrm{H}$. Tunheim for help with array processing and Maria Pla at the CSICIBMB, Barcelona, Spain for the rice samples. This work was financially supported by the European Commission through the Sixth Framework Program, integrated project Co-Extra http://www.coextra.eu;contract FOOD2005-CT-007I58) and by the Research Council of Norway (grant number 170363/D10). This is gratefully acknowledged.

\section{References}

I. James C: Global status of commercialized biotech/GM crops: 2006. Volume ISAAA Brief 35 - 2006. International Service for the Acquisition of Agri-Biotech Applications (ISAAA); 2006.

2. Macilwain C: US launches probe into sales of unapproved transgenic corn. Nature 2005, 434:423.

3. Vogel G: Genetically modified crops. Tracing the transatlantic spread of GM rice. Science 2006, 313:17|4.

4. Codex-alimentarius: Principles for the risk analysis of foods derived from modern biotechnology. 2003, CAC/GL 44: [http://www.codexalimentarius.net/download/standards/10007/ CXG 044e.pdf].

5. AgBios: GMO database. 2007 [http://www.agbios.com/dbase.php]

6. Holst-Jensen A, Rønning SB, Løvseth A, Berdal KG: PCR technology for screening and quantification of genetically modified organisms (GMOs). Anal Bioanal Chem 2003, 375(8):985-993.

7. Leimanis S, Hernandez M, Fernandez S, Boyer F, Burns M, Bruderer S, Glouden T, Harris N, Kaeppeli O, Philipp P, Pla M, Puigdomenech $P$, Vaitilingom $M$, Bertheau $Y$, Remacle J: A microarray-based detection system for genetically modified (GM) food ingredients. Plant Molecular Biology 2006, 61:123-139.

8. Rudi K, Rud I, Holck A: A novel multiplex quantitative DNA array based PCR (MQDA-PCR) for quantification of transgenic maize in food and feed. Nucleic Acids Res 2003, 3 I (I I):e62.

9. Xu J, Miao HZ, Wu HF, Huang WS, Tang R, Qiu MY, Wen JG, Zhu SF, Li Y: Screening genetically modified organisms using multiplex-PCR coupled with oligonucleotide microarray. Biosensors \& Bioelectronics 2006, 22:71-77.

10. Xu J, Zhu S, Miao H, Huang W, Fu Y, Li Y: Event-specific detection of seven genetically modified soybean and maizes using multiplex-PCR coupled with oligonucleotide microarray. Journal of Agricultural and Food Chemistry 2007, 55:5575-5579.

II. Hernandez M, Rodriguez-Lazaro D, Esteve T, Prat S, Pla M: Development of melting temperature-based SYBR Green I polymerase chain reaction methods for multiplex genetically modified organism detection. Analytical Biochemistry 2003, 323:164-170.

12. Nadal A, Coll A, La Paz JL, Esteve T, Pla M: A new PCR-CGE (size and color) method for simultaneous detection of genetically modified maize events. Electrophoresis 2006, 27:3879-3888. 
13. Mandal A, Sandgren M, Holmstrom KO, Gallois P, Palva ET: Identification of Arabidopsis thaliana sequences responsive to low temperature and abscisic acid by T-DNA tagging and in-vivo gene fusion. Plant Molecular Biology Reporter 1995, 13:243-254.

14. Baulcombe DC, Saunders GR, Bevan MW, Mayo MA, Harrison BD: Expression of biologically active viral satellite RNA from the nuclear genome of transformed plants. Nature 1986, 32I:446-449.

15. Borevitz JO, Liang D, Plouffe D, Chang HS, Zhu T, Weigel D, Berry CC, Winzeler E, Chory J: Large-scale identification of single-feature polymorphisms in complex genomes. Genome Research 2003, 13:513-523.

16. Carret CK, Horrocks P, Konfortov E, Winzeler E, Qureshi M, Newbold $C$, Ivens A: Microarray-based comparative genomic analyses of the human malaria parasite Plasmodium falciparum using Affymetrix arrays. Molecular and Biochemical Parasitology 2005, I 44: I 177-186.

17. Nesvold H, Kristoffersen AB, Holst-Jensen A, Berdal KG: Design of a DNA chip for detection of unknown genetically modified organisms (GMOs). Bioinformatics 2005, 21 : 1917-1926.

18. Lockhart DJ, Dong HL, Byrne MC, Follettie MT, Gallo MV, Chee MS Mittmann M, Wang C, Kobayashi M, Norton H, Brown EL: Expression monitoring by hybridization to high-density oligonucleotide arrays. Nature Biotechnology 1996, I 4:1675-1680.

19. Mei R, Hubbell E, Bekiranov S, Mittmann M, Christians FC, Shen MM, Lu G, Fang J, Liu WM, Ryder T, Kaplan P, Kulp D, Webster TA: Probe selection for high-density oligonucleotide arrays. Proceedings of the National Academy of Sciences of the United States of America 2003, 100: I I237- I I242.

20. Altschul SF, Madden TL, Schaffer AA, Zhang JH, Zhang Z, Miller W, Lipman D): Gapped BLAST and PSI-BLAST: a new generation of protein database search programs. Nucleic Acids Research 1997, 25:3389-3402.

21. Nielsen CR, Berdal KG, Holst-Jensen A: Characterisation of the $5^{\prime}$ integration site and development of an event-specific realtime PCR assay for NK603 maize from a low starting copy number. European Food Research and Technology 2004, 2 I 9:42 I-427.

22. Windels P, Taverniers I, Depicker A, Van Bockstaele E, De Loose M: Characterisation of the Roundup Ready soybean insert. European Food Research and Technology 2001, 213:107-1 I2.

23. Holst-Jensen A: Sampling, detection, identification and quantification of genetically modified organisms (GMOs). In Food Toxicants Analysis Edited by: Pico Y. Amsterdam, Elsevier; 2007:23I-268.

24. Dubertret B, Skourides $P$, Norris DJ, Noireaux $V$, Brivanlou $A H$, Libchaber $A$ : In vivo imaging of quantum dots encapsulated in phospholipid micelles. Science 2002, 298:1759-1762.

25. Cummins ], Ho MW, Ryan A: Hazardous CaMV promoter? Nature Biotechnology 2000, 18:363.

26. Feinberg M, Fernandez S, Cassard S, Charles-Delobel C, Bertheau $Y$ : Quantitation of 35S promoter in maize DNA extracts from genetically modified organisms using real-time polymerase chain reaction, part 2: Interlaboratory study. Journal of $A O A C$ International 2005, 88:558-573.

27. Fernandez S, Charles-Delobel C, Geldreich A, Berthier G, Boyer F, Collonnier C, Coue-Philippe G, Diolez A, Duplan MN, Kebdani N, Romaniuk M, Feinberg M, Bertheau Y: Quantification of the 35S promoter in DNA extracts from genetically modified organisms using real-time polymerase chain reaction and specificity assessment on various genetically modified organisms, part I: Operating procedure. Journal of AOAC International 2005, 88:547-557.

28. Butenko M, Patterson SE, Grini PE, Stenvik GE, Amundsen SS, Mandal A, Aalen RB: Inflorescence Deficient in Abscission controls floral organ abscission in Arabidopsis and identifies a novel family of putative ligands in plants. Plant Cell 2003, 15:2296-2307.

29. Dellaporta SL, Wood J, Hicks JB: A plant DNA minipreparation: version II. Plant Molecular Biology Reporter 1983, I:19-23.

30. Coca M, Penas G, Gomez J, Campo S, Bortolotti C, Messeguer J, Segundo BS: Enhanced resistance to the rice blast fungus Magnaporthe grisea conferred by expression of a cecropin $A$ gene in transgenic rice. Planta 2006, 223:392-406.

31. GMFF-CRL: Event specific method for the quantitation of maize line NK603 using real-time PCR. 2005 [http://gmocrl.jrc.it/summaries/NK603-WEB-Protocol\%20Validation.pdf].
32. Melchior WBJ, Von Hippel PH: Alteration of the relative stability of dA-dT and dG-dC base pairs in DNA. Proceedings of the National Academy of Sciences of the United States of America 1973, 70:298-302.

33. SALK-Institute: Salk Institute Genomic Analysis Laboratory web page. 2007 [http://signal.salk.edu/]

34. National-Center-for-Biotechnology-Information: b/2seq software. 2007 [http://www.ncbi.nlm.nih.gov/blast/b/2seq/wblast2.cgi].

35. Affymetrix: IntensityExporter software. 2007 [http:// www.affymetrix.com/support/developer/tools/devnettools.affx].
Publish with Biomed Central and every scientist can read your work free of charge

"BioMed Central will be the most significant development for disseminating the results of biomedical research in our lifetime. "

Sir Paul Nurse, Cancer Research UK

Your research papers will be:

- available free of charge to the entire biomedical community

- peer reviewed and published immediately upon acceptance

- cited in PubMed and archived on PubMed Central

- yours - you keep the copyright 